\title{
Ampliación de la distribución geográfica de Ganoderma australe (Patouillard, 1889) (Aphyllophorales-Basidiomycota), para el departamento de Cundinamarca, Colombia
}

\author{
Extension of the geographical distribution of Ganoderma australe \\ (Patouillard, 1889) (Aphyllophorales-Basidiomycota), \\ for the department of Cundinamarca, Colombia
}

\author{
César Augusto Pinzón-Osorio ${ }^{1}$, Jonás Pinzón-Osorio²
}

\begin{abstract}
Resumen
Durante 3 años se rastreó un bosque húmedo montano, ubicado en la vereda Cerca de Piedra, municipio de Chía, departamento de Cundinamarca, Colombia. Se registra una nueva localidad para Ganoderma australe. Este macromiceto se ha reportado en Colombia para los departamentos de Amazonas, Caquetá y Chocó. Se recolectaron y examinaron 32 basidiocarpos siguiendo los protocolos establecidos en la literatura especializada. Se aporta información micológica sobre características macroscópicas y microscópicas, distribución actual, época de colecta y sustrato de crecimiento de los basidiomas.
\end{abstract}

Palabras clave: Aphyllophorales, Cundinamarca, Extensión de distribución, Ganoderma australe, Micología.

\begin{abstract}
During three years we traced a wet montane forest, located in the vereda de Piedra, municipality of Chía, department of Cundinamarca, Colombia. We report a new location for Ganoderma australe. This macromycete has been reported for the departments of Amazonas, Caquetá and Chocó. We collected and examined 32 basidiocarpos following the protocols established by the specialized literature. We contributed mycological information on macroscopic and microscopic characteristics, current distribution, time of collection and substrate for growth of the basidiome.
\end{abstract}

Keywords: Aphyllophorales, Cundinamarca, Expansion of distribution, Ganoderma australe, Mycology.

\section{Introducción}

El género Ganoderma P. Karst. descrito por Karsten (1881), es el más diverso del orden Aphyllophorales Rea., con más de 300 especies (Bhosle et al. 2010). Pertenecen al Phylum basidiomycota RT Moore, clase Homobasidiomycetes RT Moore (Hibbett et al. 2007), familia Ganodermataceae Donk (Ryvarden y Johansen 1980, Schwarze y Ferner 2003).

Estos hongos lignícolas se caracterizan por presentar en el himenio poros en lugar de lamelas, basidiosporas pequeñas, con papilas irregulares y truncadas (Ryvarden y Johansen, 1980) y pared doble con ornamentación (Moncalvo y Ryvarden 1997).

En Colombia, la familia Ganodermataceae está agrupada en 4 géneros representados por 25 especies (Vasco-Palacios y Franco-Molano 2013), dentro de las que se encuentra Ganoderma australe (Fr.) Pat., un hongo leñoso, perenne, no lacado (Buchanan 2001). La especie descrita por Patouillard (1889), es también conocida como Polyporus australe Fr., Ganoderma tornatum (Pers.) Bres., y ha sido

1 Programa de Licenciatura en Biología. Herbario, Departamento de Biología, Universidad Pedagógica Nacional, Sede Bogotá, Colombia. e-mail: cesar.fungi20@gmail.com

2 Programa de Ciencias Biológicas, Departamento de Ciencias Exactas y Naturales, Universidad de Buenos Aires, Buenos Aires, Argentina. e-mail: ad10jpin@uwcad.it

Fecha recepción: Agosto 7, $2014 \quad$ Fecha aprobación: Noviembre 20, $2015 \quad$ Editor Asociado: Torres-Torres M.

(c) Rev. Biodivers. Neotrop. 2016; 6 (1): 7-11 
estudiada sobre todo por causar grandes pérdidas en la industria maderera (Karthikeyan et al. 2009) al ser agente patógeno en los bosques tropicales (Zakaria et al. 2009) y por su potencial farmacológico anticancerígeno (Trigos y Suárez-Medellín 2011).

De acuerdo con Ryvarden y Johansen (1980), G. australe se reconoce fácilmente en campo, porque presenta un píleo negro a café oscuro, opaco, himenóforo poroide color blanco-crema a gris. Las líneas resinosas que se encuentran en el contexto permiten diferenciarlo de G. applanatum (Pers.) Pat. Microscópicamente las dos especies son idénticas, sin embargo, G. australe se diferencia por presentar esporas de mayor tamaño (Ryvarden y Johansen 1980). Además, pueden llegar a variar morfológicamente por efecto del medio ambiente (Suárez-Medellín et al. 2012) mostrándose una marcada variación en la medida de las esporas, siendo en promedio mayores cuando el macrohongo prospera en zonas de gran altitud (Steyaert 1977).

A pesar que a $G$. australe se le ha reconocido una distribución mundial (Kaliyaperumal y Kalaichelvan 2008), en Colombia solo se ha reportado para los departamentos de Amazonas (Vasco-Palacios y Franco-Molano 2005), Caquetá y Chocó (Vasco-Palacios y Franco-Molano, 2013). Así, el objetivo del presente artículo es aportar datos que amplían la distribución geográfica de G. australe (Patouillard 1889), para el departamento de Cundinamarca, Colombia.
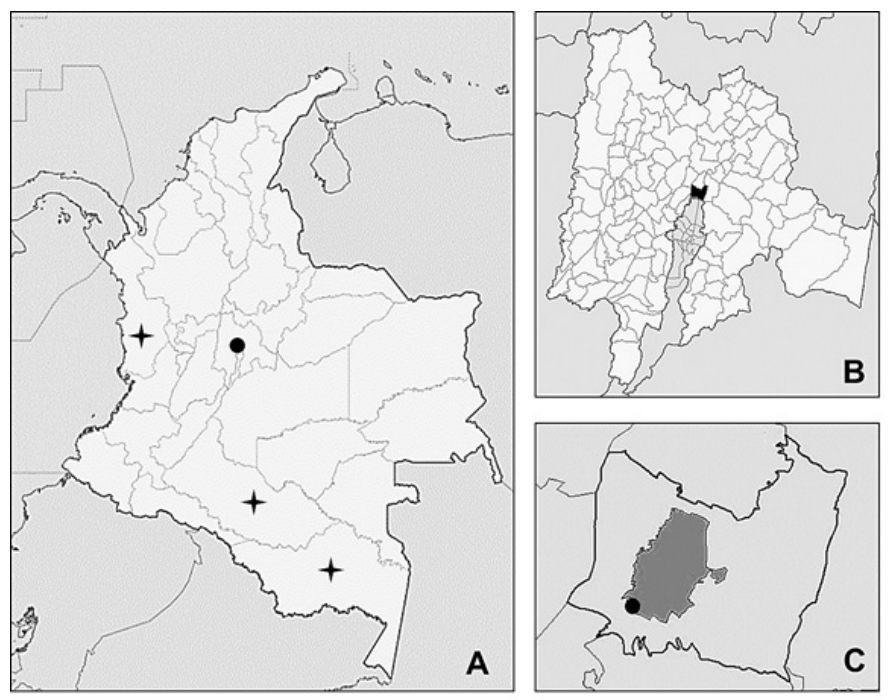

Figura 1. Distribución de Ganoderma australe para Colombia (A). Reportes previos ( + ), Amazonas (Vasco-Palacios y FrancoMolano, 2005), Caquetá y Chocó (Vasco-Palacios y FrancoMolano, 2013). Nuevo registro (0), municipio de Chía (B), vereda Cerca de Piedra (C).

\section{Metodología}

Área de estudio. La zona de muestreo se ubica en la vereda Cerca de Piedra, municipio de Chía, departamento de Cundinamarca, Colombia (Figura 1), en las coordenadas $4^{\circ}$ $50^{\prime} 53,7^{\prime}$ " $\mathrm{N}$ y $74^{\circ}$ 3' $2,8^{\prime}$ " W, sobre los 2.585 msnm, con una temperatura promedio de $14^{\circ} \mathrm{C}$. De acuerdo con la clasificación de zonas de vida propuesta por Holdridge (1971), el ecosistema evaluado corresponde a un bosque húmedo montano (bh-M) con una extensión de 4 hectáreas.

La composición vegetal general del bosque está comprendida por las familias Escalloniaceae R. Br. ex Dumort., Piperaceae Giseke., Clusiaceae L., Cunoniaceae R. Br., Betulaceae Gray., Asteraceae Bercht. y J. Presl, Rosaceae Juss., Passifloraceae L., Adoxaceae Trautv., Solanaceae Juss., Poaceae Barnhart., Ericaceae Juss., Rubiaceae Juss., Melastomataceae Juss., y Orchidiaceae Juss.

Recolección y determinación. Durante 3 años, se visitó 1 vez por mes el bosque aplicándose un muestreo de tipo oportunístico y de número fijo de troncos caídos. Los carpóforos se fotografiaron (Figura 2) y describieron macroscópicamente de forma pormenorizada siguiendo lo sugerido por Franco-Molano et al. (2005).

La evaluación microscópica (Figura 3) se realizó con un microscopio eléctrico Olympus CH-2 en el Laboratorio de la Universidad Pedagógica Nacional, sede Bogotá. En las preparaciones microscópicas se utilizó $\mathrm{KOH}$ $5 \%$ y azul de lactofenol. Para la identificación se compararon los datos con la literatura disponible (Núñez y Ryvarden 2001, Ryvarden 2004, Welti y Courtecuisse 2010, Torres-Torres et al. 2012) y se usaron las claves taxonómicas de Ryvarden y Johansen (1980) y Ryvarden (2000).

Todos los especímenes recolectados fueron acompañados de una etiqueta de referencia y se depositaron con sus respectivos datos en el Herbario de la Universidad Pedagógica Nacional, sede Bogotá (HUPN), con las iniciales ALBHMC (Aphyllophorales Lignícolas del 
bosque húmedo montano-Chía). La citación del nombre científico siguió el sistema de clasificación propuesto por Index Fungorum.

\section{Resultados}

Ganoderma australe (Fr.) Pat., Bull. Soc. mycol. Fr. 5(2,3): 65 (1889)

= Polyporus autralis Fr.: Fr., Elench. Fung. 1: 108 (1828).

= Ganoderma tornatum (Pers.) Bres., Hedwigia 53: 55 (1912).

Basidiocarpo: sésil, aplanado a ungulado, pileado, perenne. Píleo: $8.5-17 \mathrm{~cm}$ de diámetro, $10 \mathrm{~cm}$ de espesor, aumento de grosor hacia la base, semicircular, plano a dimidiado, superficie lisa, leñosa, húmeda, agrietado en zonas, opaco, glabro, marrón a café, líneas concéntricas café claro, con tintes verdosos en algunas zonas, margen entero, café en margen. Contexto: hasta $4 \mathrm{~cm}$ de grosor, pardo rojizo, manchas blancas. Hifas horizontales cercanas a los tubos, perpendiculares en la superficie del píleo, corteza de consistencia dura con $0.08 \mathrm{~cm}$ de grosor. Olor: no definido. Himenoforo: tubular, poroide, hasta 5 poros por $\mathrm{mm}$, circulares, superficie lisa, blanco vivo a crema, grisáceo crema al manipularse.

Esporada: marrón con tintes rojizos. Esporas: 6.2-13.3 x 4-7.5 $\mu \mathrm{m}, \mathrm{Q}=1.2-1-6$, elipsoidales, ornamentadas, truncadas, con columnelas, ovoides, gutuladas, con doble pared, siendo la interna ornamentada y la externa lisa, color café rojizas a amarillentas, exosporium con paredes hasta de $0.4 \mu \mathrm{m}$ de espesor. Basidios: 14-18 x 5-12 $\mu \mathrm{m}$, cilíndricos a cuadrados, espatulados, en algunos casos triangulares, 4-sterigmatados. Cistidios: no registrados. Sistema hifal: trimítico, hifas generativas fibuladas, hialinas, delgadas, 2-3 $\mu \mathrm{m}$ de grosor. Hifas esqueléticas amarillentas, paredes gruesas, 3-5 $\mu \mathrm{m}$ de ancho, algunas ramificadas a arboriformes. Hifas de unión amarillas claras, paredes delgadas tortuosas, algunas gruesas, 0.8-2.1 $\mu \mathrm{m}$ de ancho, pueden encontrarse ramificaciones delgadas de tipo arboriforme.

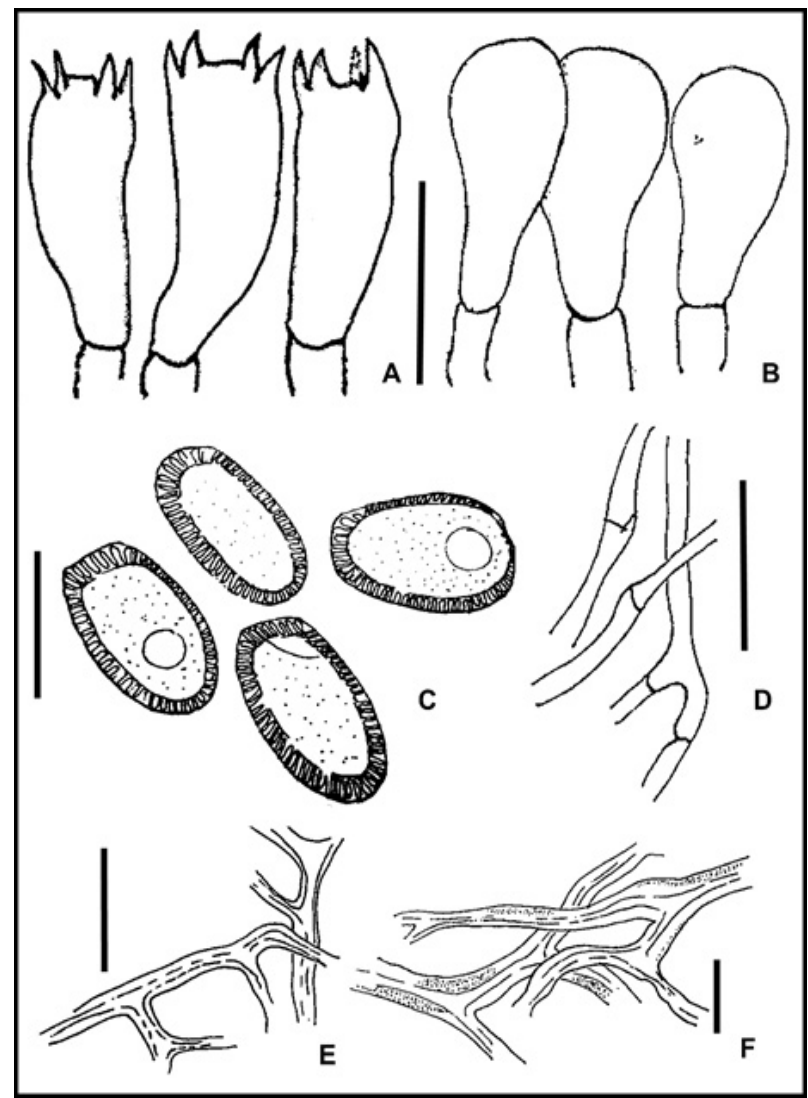

Figura 3. llustraciones microscópicas de Ganoderma australe. A. Basidio tretrasterigmatado (4) esporas, B. Basidiolos, C. Basidiosporas, D. Hifas generativas, E. Hifas de unión, F. Hifas esqueléticas. Líneas $10 \mu \mathrm{m}$. llustraciones realizadas por autor César Pinzón 
Hábito y hábitat: solitario, sobre madera de Escallonia myrtilloides L.f., y Escallonia paniculata (Ruiz y Pav) Schut., viva o muerta, en descomposición.

Ejemplares estudiados: COLOMBIA, Cundinamarca. Municipio de Chía. Vereda Cerca de Piedra. $4^{\circ}$ 50' 53,7'N, 74 5' 2,8' 'W. 2.585 msnm. 12 feb. 2012. C. Pinzón-O. 055. (HUPN). $4^{\circ} 50^{\prime} 51,04^{\prime \prime} \mathrm{N}$ y $74^{\circ} 05^{\prime}$ 04,26"W 2569 m. 18 mar. 2012. C. Pinzón-O. 065. (HUPN). $4^{\circ} 50^{\prime} 54,24^{\prime \prime} \mathrm{N}$ y $74^{\circ} 05^{\prime} 09,18^{\prime \prime} \mathrm{W} 2584$ m. 22 abr. 2012. C. Pinzón-O. 081. (HUPN). $4^{\circ} 50$ ' 52,81 '” y 74 05' 09,16"W 2572 m. 17 jun. 2012. C. Pinzón-O. 090. (HUPN). 40 50' 55,05' $\mathrm{N}$ y $74^{\circ} 05^{\prime}$ ' 07,64”W 2575 m. 29 jul. 2012. C. Pinzón-O. 105. (HUPN). $4^{\circ} 50^{\prime} 51,72$ 'N y $74^{\circ} 05^{\prime}$ '05,30'” 2570 m. 2 feb. 2013. C. Pinzón-O. 110. (HUPN). 4 50' $51,77^{\prime \prime} \mathrm{N}$ y $74^{\circ} 05^{\prime} 05,12^{\prime \prime} \mathrm{W} 2570$ m. 6 abr. 2013. $C$. Pinzón-O. 130. (HUPN). 4' 50' $52,92^{\prime}$ 'N y $74^{\circ} 05^{\prime}$ 06,01'W 2571 m. 11 ago. 2013. C. Pinzón-O. 155. (HUPN). 4 $4^{\circ} 50^{\prime} 53,81^{\prime \prime} \mathrm{N}$ y $74^{\circ} 05^{\prime} 02,28^{\prime \prime} \mathrm{W} 2568$ m. 12 oct. 2013. C. Pinzón-O. 160. (HUPN). $4^{\circ} 50^{\prime}$ 56,08'N y 74 05' 03,76'” 2570 m. 28 dic 2013. C. Pinzón-O. 179. (HUPN). $4^{\circ} 50^{\prime} 52,42^{\prime}{ }^{\prime} \mathrm{N}$ y $74^{\circ}$ 05' 02,77"W 2569 m. 23 feb. 2014. C. Pinzón-O. 190. (HUPN).

Distribución: a esta especie se le reconoce una distribución pantropical. En Colombia solo se reportaba en los departamentos de Amazonas (Vasco-Palacios y Franco-Molano 2005), Caquetá y Chocó (Vasco-Palacios y Franco-Molano 2013). El presente registro amplía su distribución para el centro del país, en el departamento de Cundinamarca, municipio de Chía, vereda Cerca de Piedra, $2.585 \mathrm{msnm}$, en un bosque húmedo montano. Teniendo en cuenta la distribución en el país, se espera que se reporte para otras zonas.

Observaciones. El presente trabajo reporta una nueva localidad para Ganoderma australe en el país, ampliándose su distribución para el departamento de Cundinamarca.

Se ha reportado que G. australe y G. applanatum tienen grandes similitudes morfológicas (Ryvarden y Johansen, 1980), lo que puede generar dificultades para su correcta diferenciación e identificación. Macroscópicamente G. australe se puede diferenciar por presentar un contexto con líneas resinosas y coloración marrón oscuro cerca de los tubos del himenóforo, aspecto que no demuestra G. applanatum. Por otra parte, el basidioma de G. australe hacia la corteza es delgado, pero G. applanatum presenta una apariencia mucho más fina con espesores menores a $0.5 \mathrm{~mm}$ (Torres-Torres et al. 2012). En este trabajo se reporta a $G$. australe con $0.08 \mathrm{~cm}$ de grosor.

Microscópicamente las dos especies son idénticas, sin embargo, G. australe se diferencia por presentar basiosporas de mayor tamaño (Ryvarden y Johansen 1980, Torres-Torres et al. 2012). Las dimensiones de este carácter en G. applanatum oscila entre 6-8.5 x 4.5-6 $\mu \mathrm{m}$ (Ryvarden y Gilbertson 1993) y 7-9.6 x 5.6-6.4 $\mu \mathrm{m}$ (Torres-Torres et al. 2012). No superan los $9.6 \mu \mathrm{m}$ de longitud, mientras que G. australe puede llegar a los $13 \mu \mathrm{m}$ (Ryvarden y Johansen 1980), aspecto determinante y que concuerda con lo reportado en este artículo, donde se alcanzan longitudes de hasta $13.3 \mu \mathrm{m}$.

La descripción y las medidas de las estructuras del espécimen concuerdan con lo reportado por Ryvarden y Johansen (1980), excepto por el diámetro de las hifas esqueléticas aquí reportadas de 3-5 $\mu \mathrm{m}$ de ancho, siendo menor al descrito por los mismos autores, de $6 \mu \mathrm{m}$.

Las dimensiones de las basidiosporas coinciden muy bien con Ryvarden y Johansen (1980), 6-13 x 4.5-8 $\mu \mathrm{m}$, al no exceder el límite inferior y superior. Sin embargo, este comportamiento no se cumple con Torres-Torres et al. (2012), 8.4-12 x 6-7.2 $\mu \mathrm{m}$. Esto se puede deber a que las medidas de las esporas pueden ser mayores cuando el macrohongo prospera en zonas de gran altitud (Steyaert 1977). Las características microscópicas consideradas como diagnósticas, varían de acuerdo con fenómenos ambientales donde el macromiceto se desarrolla (Suárez-Medellín et al. 2012).

G. australe prolifera en variedad de especies vegetales (Karthikeyan et al. 2009), mostrando un comportamiento generalista. De acuerdo con lo observado durante los tres años de rastreo, se sugiere que la especie tiene alta especificidad por el género Escallonia sp. Mutis ex L.f. como sustrato, mostrando alta dependencia a E. myrtilloides y E. paniculata para la captación de nutrientes. Lo anterior podría ser un aspecto determinante para el mantenimiento y conservación del bosque, al conocerse que este macromiceto juega un papel ecológico relevante, al dinamizar la liberación y el reciclaje de nutrientes en el proceso de descomposición de detritos (Ruiz y Varela 2006). Por tanto permite contribuir con el 
mantenimiento de la estructura y función del ecosistema forestal a través de su actividad saprofítica sobre la madera. Sin embargo, para verificar a cabalidad esta relación de especificidad hongo-planta, se requiere extender los períodos de estudio, evaluando los nutrientes aportados por el sustrato, luminosidad, edad, composición y estructura de la vegetación que compone la zona.

\section{Agradecimientos}

Los autores agradecen a la Licenciada Diana Castaño y a Nubia Ladino Ospina, Directora del Herbario de la Universidad Pedagógica Nacional, por los comentarios críticos al manuscrito.

\section{Literatura citada}

Bhosle S, Ranadive K, Bapat G, Garad S, Deshpande G, Vaidya J. 2010. Taxonomy and diversity of Ganoderma from the Western parts of Maharashtra (India). Mycosphere. 1 (3): 249-62.

Buchanan PK. 2001. A taxonomic overview of the genus Ganoderma with special references to species of medicinal and neutriceutica 1 importance. Proceedings of the International Symposium Ganoderma Science, Auckland, New Zealand, 27-29.

Franco-Molano AE, Vasco-Palacios AM, López-Quintero CA, Boekhout T. 2005. Macromicetes de la región del Medio Caquetá. Guía de campo. Medellín: Universidad de Antioquia; $211 \mathrm{pp}$.

Hibbett DS, Binder M, Bischoff JF, Blackwell M, Cannon PF, Eriksson OE, et al. 2007. A higher-level phylogenetic classification of the Fungi. Mycol Res. 111: 509-47.

Holdridge LR, Grenke W, Hatheway WH, Liang T, Tosi JA. 1971. Forest environments in tropical life zones: A pilot study. Oxford: Pergamon Press; 747 pp.

Kaliyaperumal M, Kalaichelvan PT. 2008. Ganoderma australe from southern India. Microbiol Res. 163: 286-92.

Karsten PA. 1881. Enumeratio Boletinearum et Polyporearum Fennicarum, Systemate novo dispositarum. Rev Mycol Toul. 3: 1-19.

Karthikeyan B, Joe MM, Jaleel CA. 2009. Response of some medicinal plants to Vam inoculations. J Sci Res. 1 (1): 381-6.
Moncalvo JM, Ryvarden L. 1997. A nomenclatural study of the Ganodermataceae Donk. Synopsis Fungorum. Fungiflora. Oslo, Norway; 114 pp.

Núñez M, Ryvarden L. 2001. East Asian polypores: Polyporaceae s. lato. Synopsis Fungorum. 14: 1-353.

Patouillard NT. 1889. Le genre Ganoderma. Bull Soc Mycolo France. 5 (11): 64-80.

Ruiz A, Varela A. 2006. Nuevos registros de Aphyllophorales (Basidiomicota) en bosque montano húmedo y de niebla de Colombia. Caldasia. 28 (2): 259-66.

Ryvarden L. 2000. Studies in Neotropical polypores 2: a preliminary key to neotropical species of Ganoderma with a laccatepileus. Mycologia. 92 (1): 180-91.

Ryvarden L. 2004. Neotropical Polypores. Part 1. Introduction, Hymenochaetaceae and Ganodermataceae. Synopsis Fungorum. 19: 1-227.

Ryvarden L, Johansen I. 1980. A preliminary polypores flore of East Africa. Oslo: Fungiflora; $636 \mathrm{pp}$.

Ryvarden L, Gilbertson RL. 1993. European polypores. Part 1. Synopsis Fungorum. 6: 1-387.

Schwarze F, Ferner D. 2003.Ganoderma on trees-differentiation of species and studies of invasiveness. Arboricult J. 27 (1): $59-77$.

Steyaert RL, 1977. Basidiospores of two Ganoderma species and others of two related genera under scanning electron microscope. Kew Bull. 31 (3): 437-42.

Suárez-Medellín J, Luna-Rodríguez M, Mendoza G, Salinas A, Suárez M, Trigos Á. 2012. Caracterización morfológica y molecular de una cepa del género Ganoderma. Rev Mex Mic. 36: 33-9.

Torres-Torres MG, Guzmán-Dávalos L, Gugliotta, AM. 2012. Ganoderma in Brazil: known species and new records. Mycotaxon. 121: (40) 93-132.

Trigos Á, Suárez-Medellín J. 2011. Biologically active metabolites of the genus Ganoderma: Three decades of myco-chemistry research. Rev Mex Mic. 34: 63-83.

Vasco-Palacios AM, Franco-Molano AE. 2013. Diversity of Colombian Macrofungi. (Ascomycota-Basidiomycota). Mycotaxon. 121: 1-58.

Vasco-Palacios AM, Franco-Molano AE, López-Quintero C, Boekhout T. 2005. Macromicetes (Ascomycota) de la región del Medio Caquetá, departamento de Caquetá y Amazonas (Colombia). Biota Colomb. 6 (1): 127-40.

Welti S, Courtecuisse R. 2010. The Ganodermataceae in the French West Indies (Guadeloupe and Martinique). Fungal Divers. 43 (1):103-26.

Zakaria L, Ali N, Salleh B, Zakaria M. 2009. Molecular analysis of Ganoderma species from different hosts in peninsula Malaysia. J Biol Sci. 9 (1):12-20. 